\title{
Malaria parasite tyrosyl-tRNA synthetase secretion triggers pro-inflammatory responses
}

\author{
Tarun Kumar Bhatt ${ }^{1, \star}$, Sameena Khan ${ }^{1, \star}$, Ved Prakash Dwivedi², Mudassir Meraj Banday ${ }^{1}$, Arvind Sharma', \\ Anmol Chandele1, Noelia Camacho ${ }^{3}$, Lluís Ribas de Pouplana ${ }^{4}$, Yang Wu ${ }^{5}$, Alister G. Craig 5 , \\ Antti Tapani Mikkonen ${ }^{6}$, Alexander Gerd Maier ${ }^{6}$, Manickam Yogavel' \& Amit Sharma ${ }^{1}$
}

Malaria infection triggers pro-inflammatory responses in humans that are detrimental to host health. Parasite-induced enhancement in cytokine levels correlate with malariaassociated pathologies. Here we show that parasite tyrosyl-tRNA synthetase (PfTyrRS), a housekeeping protein translation enzyme, induces pro-inflammatory responses from host immune cells. PfTyrRS exits from the parasite cytoplasm into the infected red blood cell (iRBC) cytoplasm, from where it is released into the extracellular medium on iRBC lysis. Using its ELR peptide motif, PfTyrRS specifically binds to and internalizes into host macrophages, leading to enhanced secretion of the pro-inflammatory cytokines TNF- $\alpha$ and IL-6. PfTyrRS-macrophage interaction also augments expression of adherence-linked host endothelial receptors ICAM-1 and VCAM-1. Our description of PfTyrRS as a parasite-secreted protein that triggers proinflammatory host responses, along with its atomic resolution crystal structure in complex with tyrosyl-adenylate, provides a novel platform for targeting PfTyrRS in anti-parasitic strategies.

\footnotetext{
${ }^{1}$ Structural and Computational Biology Group, International Centre for Genetic Engineering and Biotechnology (ICGEB), Aruna Asaf Ali Road, New Delhi 110 067, India. ${ }^{2}$ Immunology Group, International Centre for Genetic Engineering and Biotechnology (ICGEB), Aruna Asaf Ali Road, New Delhi 110067, India. ${ }^{3}$ Institute for Research in Biomedicine (IRB), C/ Baldiri Reixac 15-21, 08028 Barcelona, Catalonia, Spain. ${ }^{4}$ Catalan Institution for Research and Advanced Studies (ICREA), Passeig Lluís Companys 23, 08010 Barcelona, Catalonia, Spain. ${ }^{5}$ Liverpool School of Tropical Medicine, Pembroke Place, Liverpool L3 5QA, UK. ${ }^{6}$ Department of Biochemistry, La Trobe Institute for Molecular Science, La Trobe University, Melbourne Victoria 3086, Australia. *These authors contributed equally to this work. Correspondence and requests for materials should be addressed to A.S. (email: amit.icgeb@gmail.com).
} 
A minoacyl-tRNA synthetases are ancient enzymes responsible for genetic code translation ${ }^{1}$. These ubiquitous enzymes attach amino acids onto cognate transfer RNAs during protein translation, and are divided into two classes based on their structural topology $y^{1,2}$. Class I synthetases, like tyrosyl-tRNA synthetase (TyrRS), are characterized by a Rossmann fold, and this class contains two conserved ATP-binding motifs (HIGH and KMSKS $)^{3,4}$. Apart from their parent aminoacylation activity, several aminoacyl-tRNA synthetases perform non-canonical functions in transcriptional regulation, apoptosis, ribosomal RNA biogenesis, angiogenesis and cell signalling ${ }^{5-8}$. Human TyrRS is processed by an elastase enzyme yielding an amino-terminal TyrRS (also known as mini-TyrRS) and carboxy-terminal EMAPII-like domain ${ }^{9-13}$. The latter contains cell signalling activity whereas human mini-TyrRS mimics interleukin-8 (IL-8) to chemo-attract polymorphonuclear cells ${ }^{9-13}$. Human mini-TyrRS interacts with CXCR1/2 receptors and acts as an angiogenic cytokine using a motif called ELR (Glu-LeuArg) that it shares with IL-8 (Fig. 1a). For the human mini-TyrRS ELR motif to be functional, the intact human enzyme is required to undergo proteolytic cleavage, which results in the release of EMAPII domain and subsequent exposure of the ELR motif ${ }^{9-13}$.

Plasmodium parasites are the causative agents of malaria. It is estimated that $>300$ million clinical malaria cases occur annually, resulting in $>1$ million deaths ${ }^{14}$. Malaria-related deaths occur owing to a range of syndromes including acute anemia and/or cerebral/ placental malaria ${ }^{14,15}$. Most malaria pathology results from an acute inflammatory spurt, followed by activation of the host immune system and dysregulated cytokine release ${ }^{14-18}$. Human patient studies and mouse malaria models have consistently suggested that infection by the malaria parasite triggers an increase in the levels of numerous pro-inflammatory agents including tumour necrosis factor (TNF- $\alpha$ ), interferon gamma (IFN- $\gamma$ ) and interleukin 6 (IL-6) in blood plasma ${ }^{19-21}$. Although pro-inflammatory cytokines have a major role in patho-physiology of malaria disease ${ }^{16-21}$, the molecular mechanisms of cytokine release from immune cells remains largely unclear. Until now, several parasite-derived molecular agents have been implicated in triggering cytokine release $\mathrm{e}^{22-25}$, but parasitesecreted protein(s) that potentially trigger specific cytokine secretion from host immune cells are less well understood. It is critical to understand the pathways and mechanisms that lead to cytokine release in malaria as disease pathology, directly or indirectly, generally results from dysregulation of circulating TNF- $\alpha$ and IL-6 amounts ${ }^{22-25}$.

Plasmodium falciparum genome contains two copies of tyrosyltRNA synthetase-tyrosyl-tRNA synthetase 1 (hereafter PfTyrRS, Plasmodb ID = MAL8p1.125) and tyrosyl-tRNA synthetase 2 (hereafter PfTyrRS ${ }^{\text {api }}$, Plasmodb ID = PF11_0181). Pf TyrRS was predicted to be cytoplasmic whereas PfTyrRS ${ }^{\text {api }}$ is probably targeted to the parasite apicoplast ${ }^{6}$. Here we have investigated the structural, immunological and spatial localization attributes of PfTyrRS. Our comprehensive analyses provide a platform for focusing on PfTyrRS as a new target for development of novel malaria intervention strategies.

\section{Results}

PfTyrRS characterization and structure. Bacterially overexpressed PfTyrRS was purified to homogeneity using affinity and gel-filtration chromatography, and the protein migrates as a dimer (Fig. 1b). PfTyrRS enzyme activity was confirmed using tRNAs obtained by in vitro transcription using T7 RNA polymerase (Fig. 1c) ${ }^{26-28}$. We determined the three-dimensional structure of PfTyrRS in complex with YAP (tyrosyl-adenylate) to a resolution of $2.2 \AA$ (Fig. 2a,b; Table 1). The crystallographic asymmetric unit contains a canonical tyrosyl-tRNA synthetase dimer. Eighteen N-terminal and three C-terminal residues were not clearly defined in the electron density for each monomer. The overall fold of PfTyrRS is typical of class I synthetases $^{3,4}$ and comprises a catalytic domain (residues 18-260,
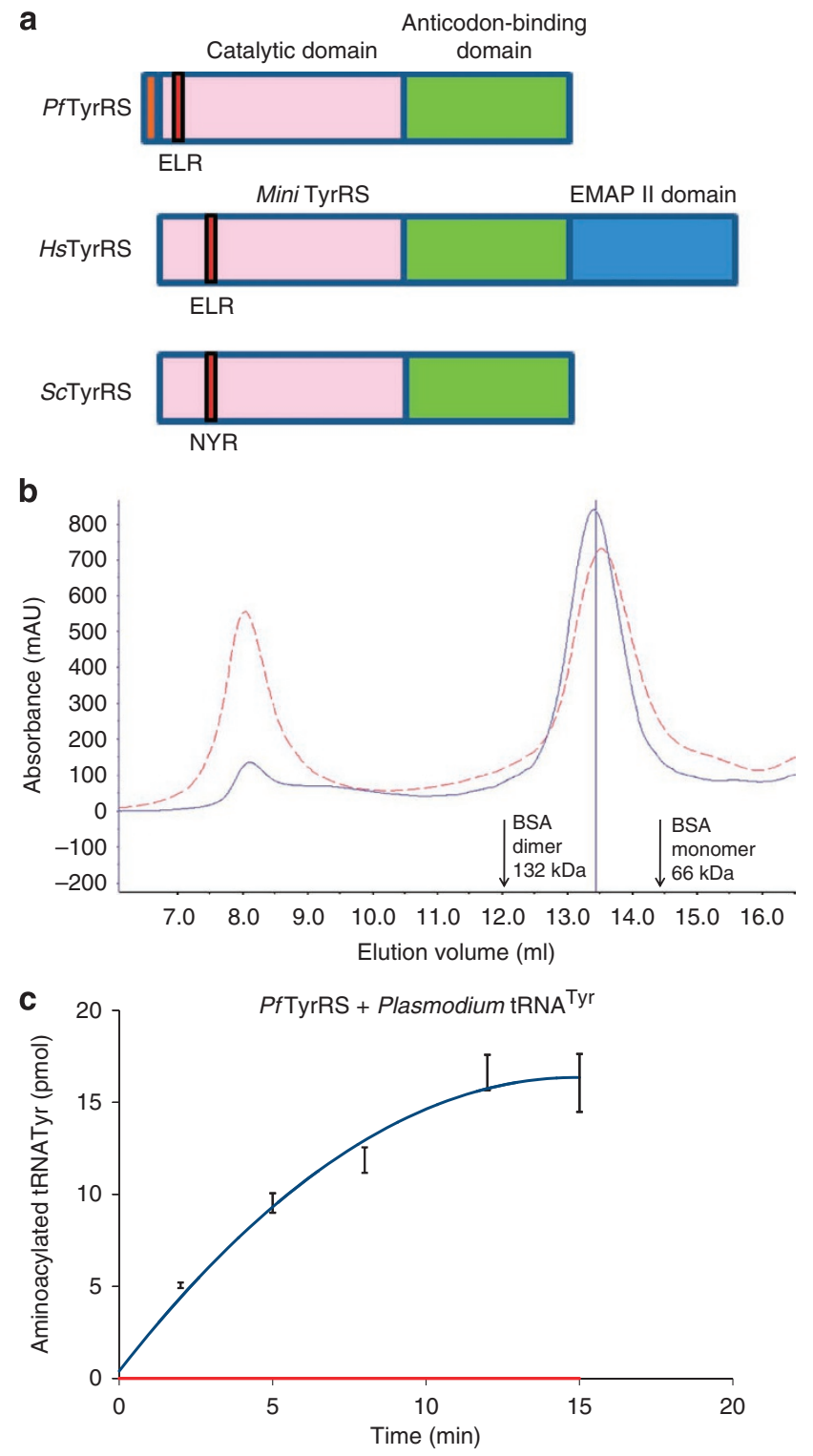

Figure 1 | Tyrosyl tRNA synthetase domain organization and enzyme characterization. (a) Domain organization of TyrRS from P. falciparum, human and yeast. The 21 residue $\mathrm{N}$-terminal extension in PfTyrRS is shown in orange. The ELR motif is shown in red and in yeast TyrRS it is replaced by NYR. (b) Gel filtration curves show both wild-type PfTyrRS (solid blue line) and ELR/AAA mutant (dotted red line) proteins as dimers in solution. (c) Aminoacylation curves with (blue line) and without (red line) added native PfTyrRS and using Pf-tRNA ${ }^{\text {Tyr }}$.

Rossmann fold) and an anti-codon-binding domain (residues 261-370). Human mitochondrial and malaria parasite apicoplastic TyrRS belong to bacterial lineage, whereas human cytoplasmic TyrRS and PfTyrRS belong to a separate eukaryotic group ${ }^{6,29}$ (Fig. 2c). Structural superposition of $292 \mathrm{C}^{\alpha}$ atoms between PfTyrRS and human mini-TyrRS (Protein Data Bank, accession code 1N3 L) gives an RMSD of $\sim 1.9 \AA$ (sequence identity $~ 30 \%$ ), whereas the RMSD between $144 \mathrm{C}^{\alpha}$ atoms of PfTyrRS and mitochondrial HsTyrRS (PDB: 2PID) is $\sim 2.5 \AA$ (sequence identity $<10 \%$ ). In PfTyrRS, the solvent accessible buried surface area at dimer interface is $\sim 1,337 \AA^{2}$, and its value is comparable with L-Tyr bound mini-HsTyrRS $\left(1,380 \AA^{2}\right)$ but not with the unliganded miniHsTyrRS (buried area of $1,531 \AA^{2}$ ). In mini-HsTyrRS structure ${ }^{13}$, the adenosine binding pocket is empty and seems enlarged by 
a

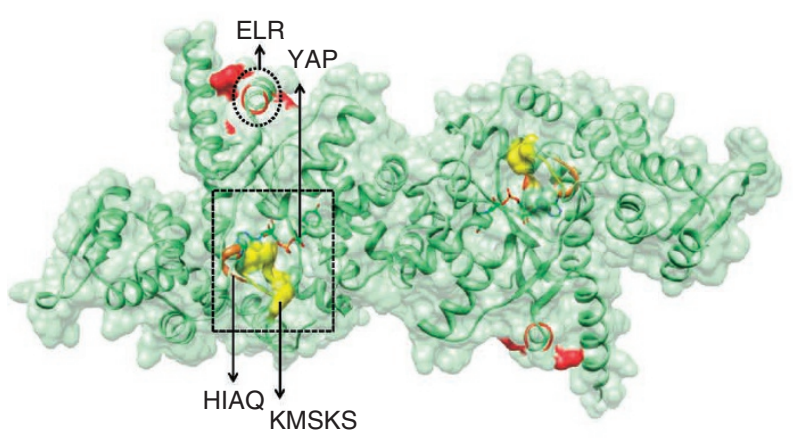

b

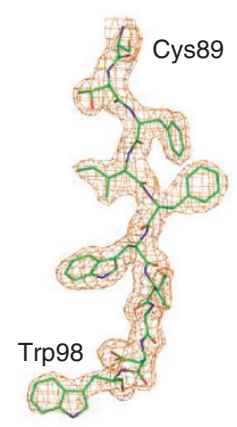

d

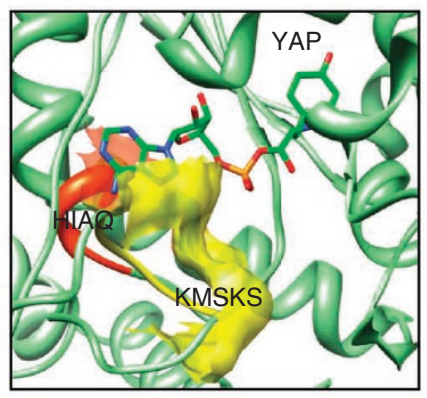

PfTyrRS

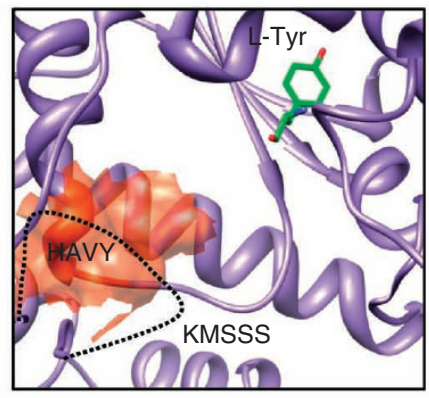

Cytoplasmic mini-HsTyrRS
C
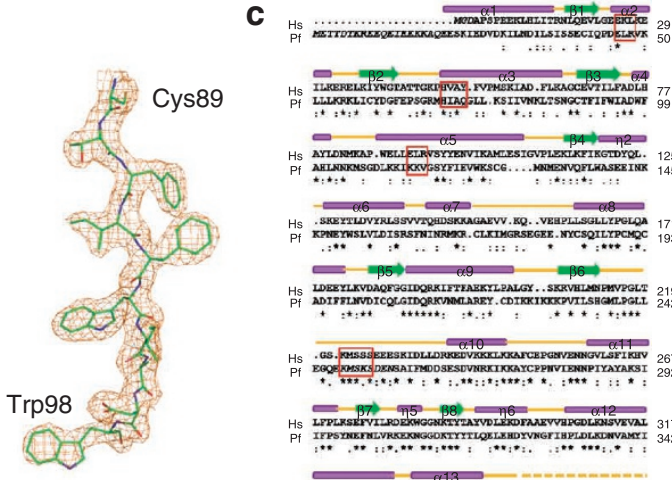

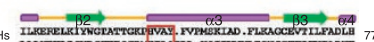

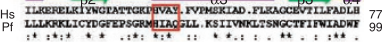

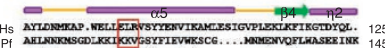

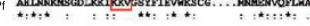

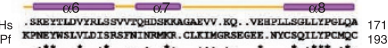

E-

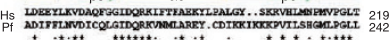

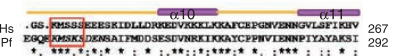

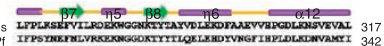

Is

e

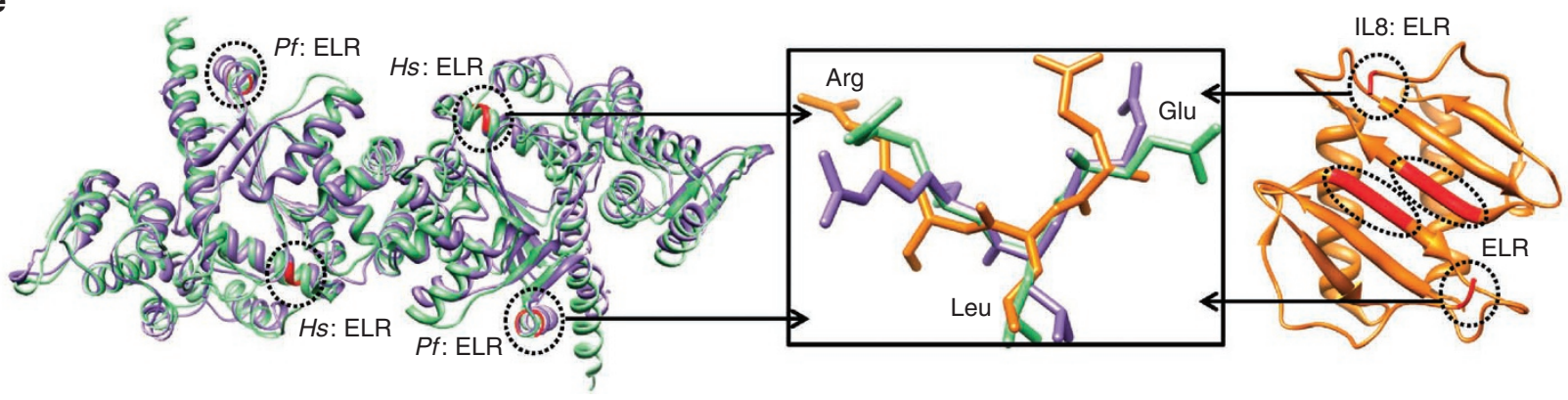

Figure 2 | Crystal structure of PfTyrRS. (a) PfTyrRS:Tyr-AMP (YAP) complex structure where the ELR, KMSKS and HIGH motifs are shown in red, yellow and orange, respectively. Bound YAP is shown in stick model (green). (b) Stereoview of the experimental electron density map (2|Fo|-|Fc|) for a portion of the final atomic model. The map is contoured at $1.2 \sigma$ level. (c) Sequence alignment between mini-HsTyrRS and PfTyrRS. Important motifs are highlighted in red boxes and the ELR motifs are marked. (d) A close-up view of KMSKS loop and active site region of PfTyrRS along with corresponding regions from cytoplasmic mini-HsTyrRS and mitochondrial HsTyrRS (with 5'-O-[N-(L-Tyrosyl) sulfamoyl] adenosine also known as YSA). The disordered KMSKS loop is shown as dotted lines in human structures and the bound substrate/analog is shown as sticks. (e) Structural alignment and ELR motifs in mini-HsTyrRS (purple), PfTyrRS (green) and HsIL-8 (orange).

$\sim 3 \AA$ on either side of the binding pocket whereas, in YSA (5'-O[N-(L-Tyrosyl) sulfamoyl] adenosine)-bound mitochondrial HsTyrRS ${ }^{29}$ and in YAP-bound PfTyrRS, the adenosine-binding pocket is tighter (Fig. 2d). In PfTyrRS, the key synthetase motif of KMSKS loop is highly ordered and close to the bound substrate at the active site, whereas, in both cytoplasmic and mitochondrial HsTyrRS, this is found to be disordered (Fig. 2d). We find several other key structural differences between mini$H s$ TyrRS and PfTyrRS (Fig. 2c,d). First, at the primary sequence level, $P f$ TyrRS has an $\mathrm{N}$-terminal 21 residue extension whose role is unclear (Fig. 1a). On the other hand, human cytoplasmic TyrRS has a C-terminal extension in the form of an EMAPII domain that undergoes proteolytic processing to reveal a host module more similar in size to $P f \operatorname{TyrRS}^{9-13}$ (Fig. 1a). The most notable and intriguing feature of both the mini-HsTyrRS and PfTyrRS is presence of an ELR motif (Figs. 1a and 2e). This tri-peptide signature is present in, among others, cytokines like IL-8 (refs 9-13,30). As shown in Figure 2e, ELR motifs decorate the surfaces of IL-8, mini-HsTyrRS and PfTyrRS. Structural alignment of ELR motifs from these three reveals striking conformational conservation (RMSD $<1 \AA$ ). In PfTyrRS, the ELR motif resides on $\alpha$-helix 2 whereas, in mini-HsTyrRS, it is located at $\alpha$-helix 5 (Fig. 2e). In both PfTyrRS and mini-HsTyrRS, the ELR motif is significantly distal from aminoacylation sites (Fig. 2e). In full-length cytoplasmic HsTyrRS, the ELR motif is buried under the C-terminal EMAPII domain, and this motif is exposed after proteolytic cleavage ${ }^{9-13}$. In contrast, the PfTyrRS ELR motif is exposed in three-dimensional space and does not require processing for its surface display. Therefore, in the parasite enzyme the ELR tri-peptide is continually solvent accessible owing to its presence in a different part of the overall three-dimensional structure relative to human TyrRS (Fig. 2a,e). Subtle structural perturbations caused by single residue mutations near the ELR motif can activate procytokine activity of human TyrRS ${ }^{11}$. These data on human TyrRS ${ }^{11}$ suggested that the twin attributes of cytokine trigger and 


\begin{tabular}{|c|c|}
\hline Parameters & PfTyrRS-YAP \\
\hline \multicolumn{2}{|l|}{ Data collection } \\
\hline Space group & $\mathrm{C} 2$ \\
\hline \multicolumn{2}{|l|}{ Unit cell dimensions } \\
\hline$a, b, c(\AA)$ & $137.5,46.5,141.3$ \\
\hline$\alpha, \beta, \gamma\left(^{\circ}\right)$ & $90,93.8,90$ \\
\hline Resolution $(\AA)$ & $50.0-2.2$ \\
\hline$R_{\text {merge }}$ & $0.056(0.405)$ \\
\hline$I / \sigma(I)$ & $51.0(4.8)$ \\
\hline Completeness (\%) & $94.7(82.2)$ \\
\hline Redundancy & $14.1(9.8)$ \\
\hline \multicolumn{2}{|l|}{ Refinement } \\
\hline Resolution $(\AA)$ & $50.0-2.2$ \\
\hline No. of reflections & 42,842 \\
\hline$R_{\text {work }} / R_{\text {free }}$ & $0.172 / 0.214$ \\
\hline \multicolumn{2}{|l|}{ No. of atoms } \\
\hline Protein (two molecules) & 5,726 \\
\hline Ligand/waters & $70 / 371$ \\
\hline \multicolumn{2}{|l|}{$B$-factors $\left(\AA^{2}\right)$} \\
\hline Protein & 57 \\
\hline Ligand/waters & $39 / 61$ \\
\hline \multicolumn{2}{|l|}{ R.m.s. deviations } \\
\hline Bond lengths ( $\AA$ ) & 0.007 \\
\hline Bond angles $\left({ }^{\circ}\right)$ & 1.09 \\
\hline
\end{tabular}

Values in parentheses are for highest-resolution shell.

aminoacylation can coexist in a tyrosyl-tRNA synthetase ${ }^{11}$. Indeed, our description of the parasite PfTyrRS provides an excellent natural example of a synthetase enzyme with constitutively active ELR motif (Fig. 2). Interestingly, the ELR motif is absent in TyrRSs from lower eukaryotes and prokaryotes (Fig. 1a), again suggesting that ELR motif is independent of the parent aminoacylation activity of these enzymes.

Structural comparisons suggest sequence differences in 11 key residues that contribute to L-Tyr binding ( 5 residues) and adenosine binding ( 6 residues) between human and parasite enzymes (Fig. 2c,d). Residues in the conserved HIGH and KMSKS motifs are different between PfTyrRS (HIAQ and KMSKS) and miniHsTyrRS (HAVY and KMSSS). In the L-Tyr bound mini-HsTyrRS, a $\mathrm{K}^{+}$ion is bound near adenine binding pocket whereas this space is empty in unliganded state of mini-HsTyrRS. In PfTyrRS, there is no density for an ion like $\mathrm{K}^{+}$, and further the residues involved in $\mathrm{K}^{+}$ ion coordination in mini-HsTyrRS are not conserved in PfTyrRS. Therefore, several subtle yet crucial differences between host and pathogen tyrosyl-tRNA synthetase active sites (Fig. 2b-d) present a window for exploiting the malaria parasite enzyme for structurebased inhibitor discovery-consistent with recent high-throughput chemical library screening data ${ }^{31}$.

Parasite and erythrocyte localization of PfTyrRS. Confocal microscopy was performed using protein-A purified IgG fraction of rabbit antibodies that were raised against recombinant $P f$ TyrRS. Our localization data show that PfTyrRS is present in all three stages of asexual parasite life cycle: rings, trophozoites and schizonts (Fig. 3a; Supplementary Movies 1, 2 and 3 based on z-stacked confocal analyses of rings, trophozoites and schizonts, respectively-DAPI is in blue and PfTyrRS in green). Closer examination of PfTyrRS localization in infected erythrocytes during trophozoite and schizont stages revealed possible secretion of PfTyrRS into infected red blood cell (iRBC), despite absence of an export

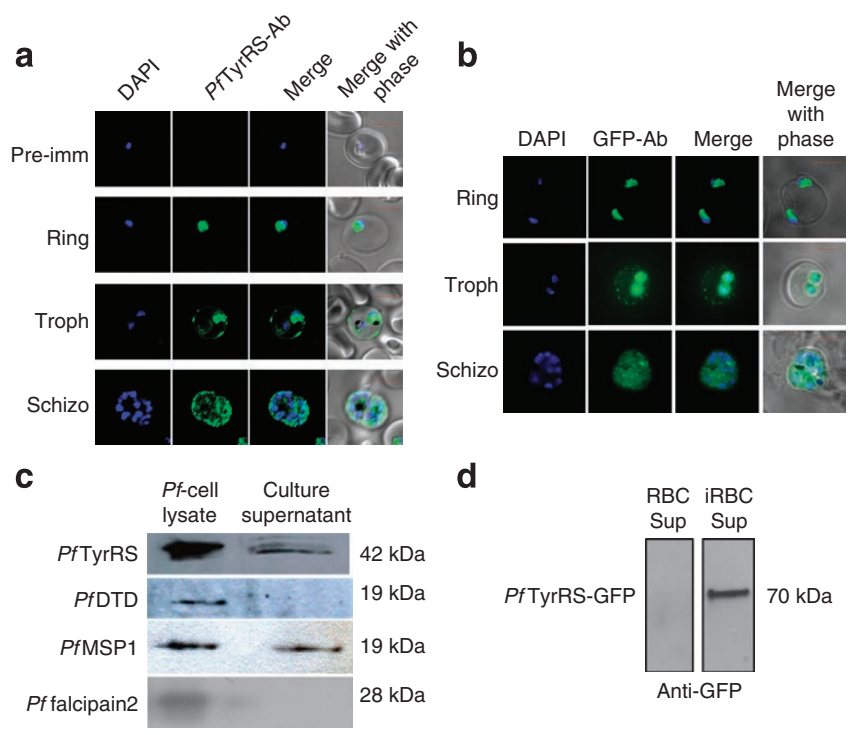

Figure 3 | Cellular localization of PfTyrRS. (a) Confocal microscopy of $P$. falciparum asexual stages-rings, trophozoites and schizonts using anti-sera raised in rabbits against $P f$ TyrRS. Scale bar in red corresponds to a size of $5 \mu \mathrm{m}$ (b) Confocal microscopy using anti-GFP antibody (green) in PfTyrRS-GFP transgenic parasite cell lines. Parasite nucleus was stained with DAPI (blue) in each case. Scale bar in red corresponds to a size of $5 \mu \mathrm{m}$. (c) Enhanced chemi-luminiscence of parasite culture supernatants using antibodies against PfTyrRS, PfDTD, PfMSP1 and Pf falcipain 2 and (d) Enhanced chemiluminiscence of parasite culture supernatants using anti-GFP antibody.

signal in PfTyrRS sequence (Fig. 3a). Similar results were obtained using PfTyrRS-GFP fusion parasite transgenic cell lines (Fig. 3b). We therefore tested whether secretion of PfTyrRS occurred from schizonts at time of iRBC rupture. To address this, culture supernatants of infected RBCs were separated on SDS-PAGE, and western blotting was performed using anti-PfTyrRS antisera (Fig. 3c). This experiment indicated a band of $\sim 42 \mathrm{kDa}$ in iRBC medium that corresponds to the molecular size of PfTyrRS. As controls, the parasite-secreted MSP1 and non-secreted proteins like DTD ${ }^{32,33}$ and falcipain 2 were also used (Fig. 3c). We also performed western blot analysis using anti-GFP antibody on PfTyrRS-GFP parasite cell line supernatants. PfTyrRS-GFP fusion protein band of expected molecular weight $(70 \mathrm{kDa})$ was clearly seen in iRBCs supernatants but not in supernatants from uninfected RBCs controls (Fig. 3d). These experiments suggest that PfTyrRS is expressed in all asexual parasite stages, and that it is exported to the host erythrocyte cytosol, from where it is released into blood plasma on iRBC rupture.

PfTyrRS interaction with host immune cells. Release of PfTyrRS from iRBCs and the presence of an exposed and conformationally competent ELR motif persuaded us to address directly the binding of TyrRS to host immune cells. We performed an in vivo experiment where mice were infected with Plasmodium yoelii (XNL). Subsequently, spleen cells containing peripheral blood mononuclear cells (PBMCs) were isolated from these infected mice and then presence of native TyrRS was tested using anti-TyrRS-specific antibodies by flow cytometry (Fig. 4a). We observed specific TyrRS binding to mouse macrophages and dendritic cells, among others (Fig. 4a). A corresponding in vitro experiment using human PBMCs also showed specific interaction of PfTyrRS with human macrophages and, to a lesser degree, with dendritic cells (Fig. 4b). To validate these observations further, we assessed binding of PfTyrRS to mouse and human immune cells using confocal microscopy (Fig. 4c,d). We detected both binding and internali- 

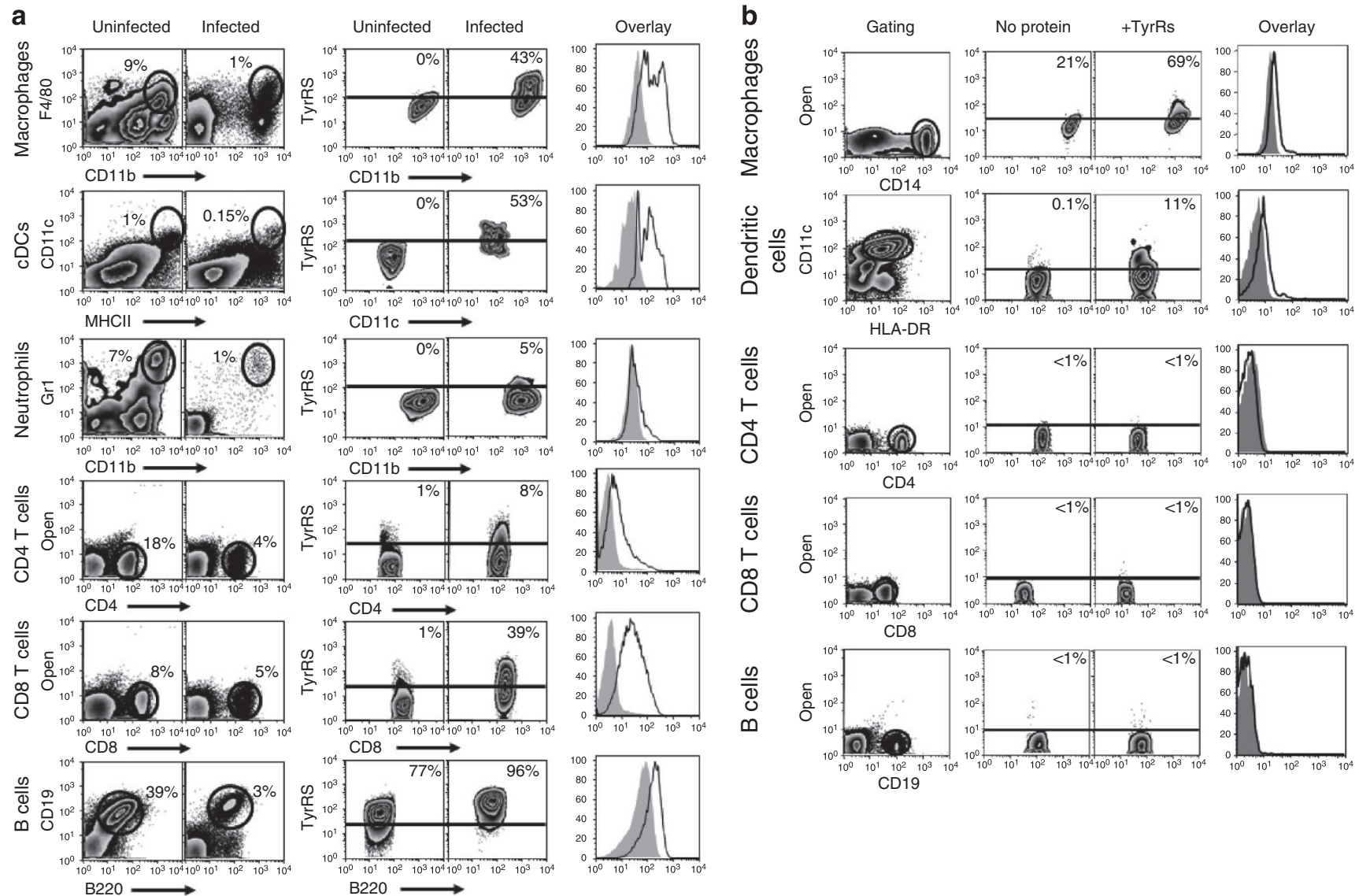

。

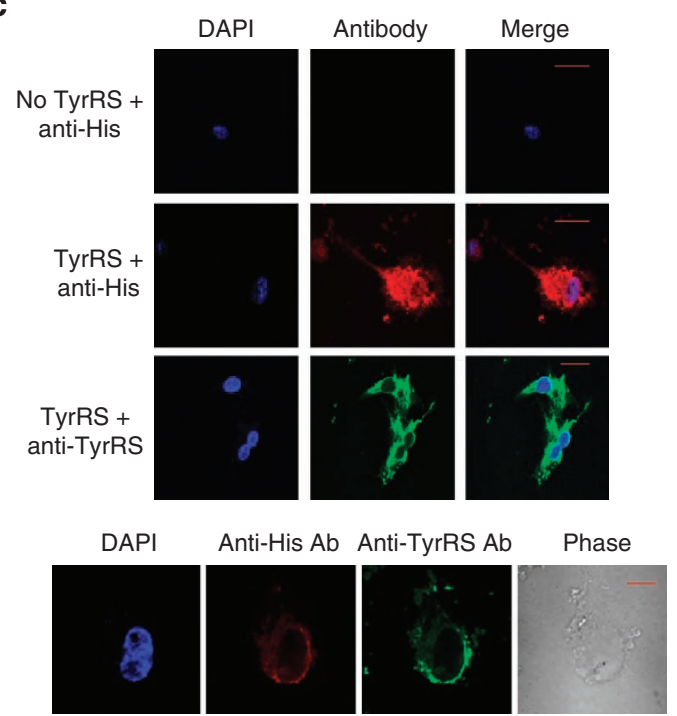

d

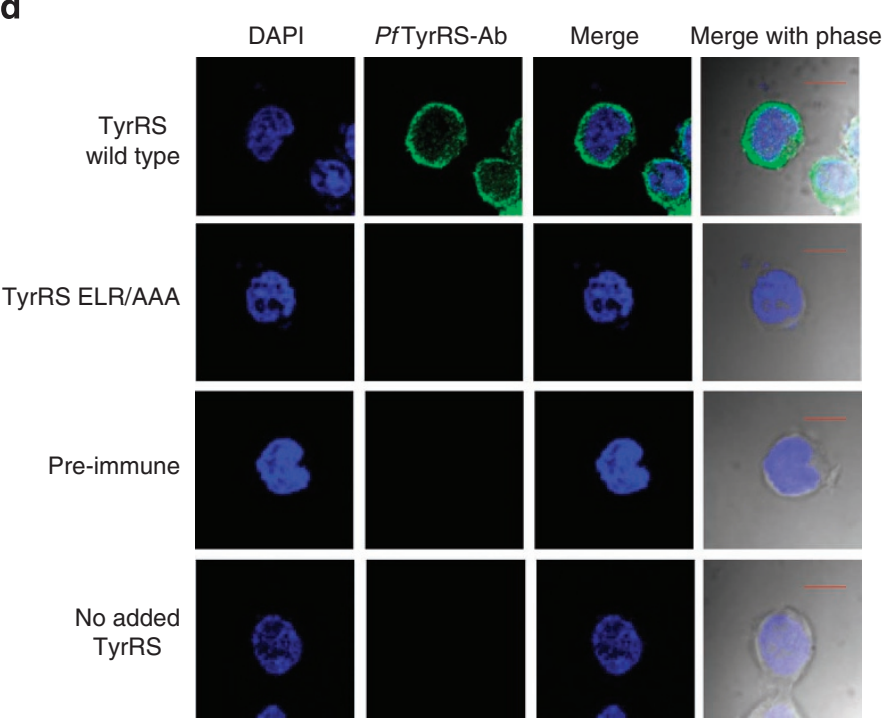

Figure 4 | TyrRS interaction with PBMCs in vivo and in vitro. (a) FACS-based binding of mouse PBMCs with anti-PfTyrRS antibodies in an ex vivo experiment. Left panel shows gating; middle panel shows TyrRS binding in immune cells tested in both uninfected and infected mice. Overlay histograms are derived from the TyrRS expression dot plots wherein solid grey histogram is uninfected and the black bold line represents data for infected mice. Data are cumulative of two independent experiments. (b) Binding of PfTyrRS to human PBMCs showing specific interaction with human macrophages and dendritic cells. Left panel shows gating; middle panel shows PfTyrRS binding to various immune cells. The overlay histogram is derived from PfTyrRSbinding dot plots wherein solid grey histogram is without PfTyrRS and black solid line is with PfTyrRS. Confocal images of binding and internalization of PfTyrRS into (c) mouse dendritic cells (top panel), mouse macrophages (bottom panel) and (d) human macrophages. In (d) along with wild-type PfTyrRS (green), the controls of pre-immune sera, ELR/AAA mutant PfTyrRS, and primary/secondary antibodies alone (that is, no added PfTyrRS) are shown. For nucleus visualization, DAPI (blue) localization is also shown. The red scale bar in confocal images corresponds to a size of $15 \mu \mathrm{m}$.

zation of PfTyrRS by host macrophages. Supplementary Movie 4 is based on Z-stacking of these confocal images where macrophages are stained for nucleus (DAPI, blue) and for PfTyrRS (in green). These data led us to hypothesize an ELR-motif-mediated interaction of $P$. falciparum tyrosyl-tRNA synthetase with human macrophages (Fig. 4c,d). 
Triggering of cytokine secretion by PfTyrRS. Our observations on interaction of PfTyrRS with host immune cells prompted us to test whether the ELR motif present in PfTyrRS could enable it to trigger release of cytokines from macrophages. We tested this hypothesis by incubating mouse and human macrophage cell lines with recombinant wild-type PfTyrRS, with a mutant ELR/AAA-PfTyrRS, with lipopolysaccharide (LPS) (as positive control), with media alone and PfDTD (last two as negative controls). In these studies, the amounts of PfTyrRS used were in nanomolar (nM) concentration ranges, similar to amounts used in studies with human mini-TyrRS ${ }^{9-13}$. Time kinetic analysis using ELISA revealed maximal production of the inflammatory cytokines TNF- $\alpha$, IL- 6 and IL- $1(\alpha$ and $\beta)$ within 6 hours of culture (Tukey's test $-P<0.001$ ) (Fig. 5a,b), but not of IL-10 or IL-12 (Fig. 5c). The cytokine release mediated by PfTyrRS

a

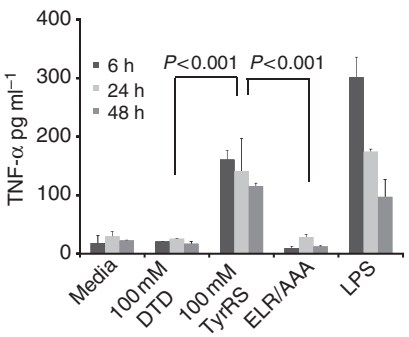

b

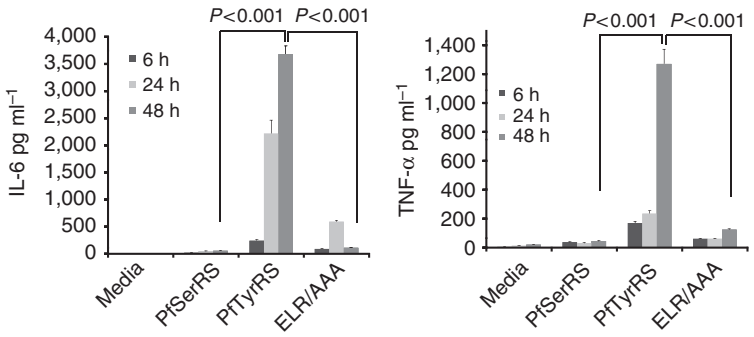

C

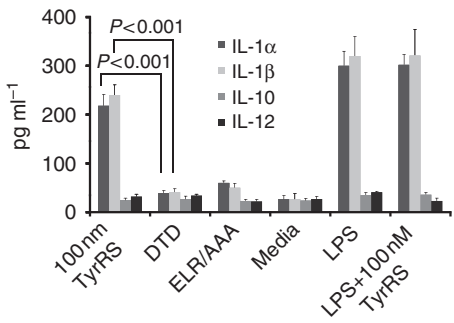

\section{d}
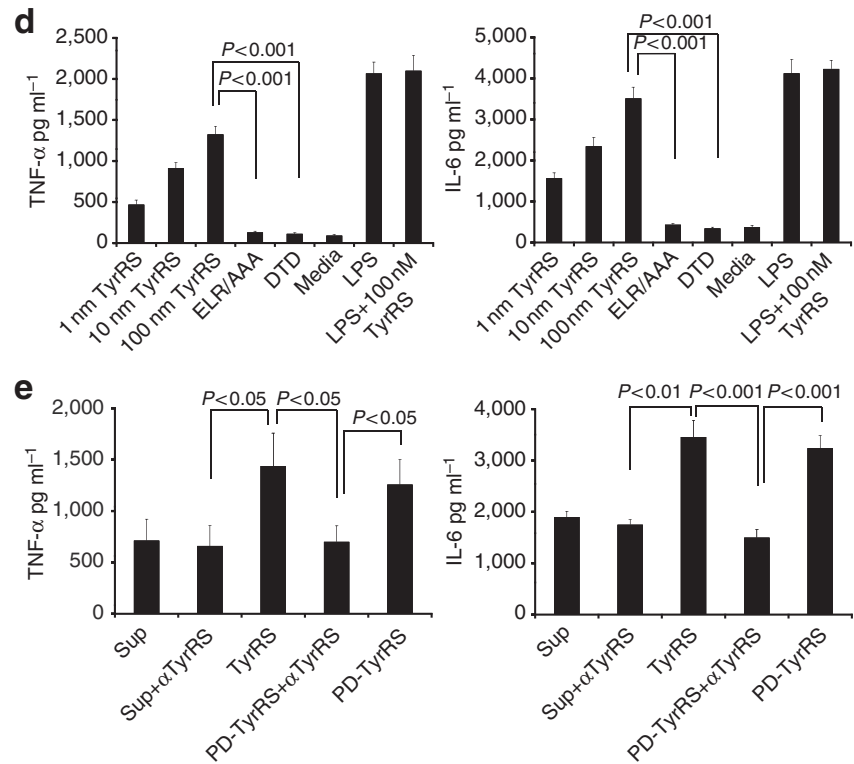

was dose-dependent, and occurred as early as 4 hours post-incubation of macrophages with PfTyrRS (Fig. 5d). Interestingly, the mutant PfTyrRS (where ELR was replaced with AAA sequence) did not trigger cytokine release from macrophages and behaved similar to the control proteins PfDTD ${ }^{32,33}$ or PfSerRS. In all experiments, LPS was used as a positive control, and all proteins used in these experiments were confirmed to be endotoxin free (Methods). We also observed that PfTyrRS that had been immunoprecipitated from parasite culture supernatants was equally capable of triggering release of TNF- $\alpha$ and IL- 6 from human macrophage ThP1 cell lines (Fig. 5e). Finally, pre-incubation of PfTyrRS with anti-PfTyrRS antibodies substantially blocked TNF- $\alpha$ (Tukey's test $-P<0.05)$ and IL-6 production (Tukey's test $-P<0.01$ ) from human macrophages (Fig. 5e), suggesting specific, motif-based PfTyrRS interaction with human macrophages.

Potential receptor(s) for PfTyrRS on human macrophages. To identify a receptor for PfTyrRS on macrophages, we pre-incubated human macrophages with anti-CXCR1/2 and anti-PfTyrRS antibodies, and tested whether antibody-mediated receptor or protein blockades inhibited PfTyrRS-macrophage interaction (Fig. 6a) Specific antibody-mediated blocking of CXCR2 receptor (Tukey's test $-P<0.05$ ), but not the CXCR1 receptor, on human macrophages reduced PfTyrRS binding by $\sim$ twofold (Fig. 6a). Blocking of PfTyrRS with anti-PfTyrRS antibodies also reduced binding of the protein to macrophages (Tukey's test $-P<0.05$ ) (Fig. 6a). Furthermore, blockage of PfTyrRS-macrophage interactions using either anti-CXCR2 or anti-PfTyrRS antibodies also significantly reduced the release of TNF- $\alpha$ (Tukey's test $-P<0.05$ ) and IL- 6 (Tukey's test$P<0.001$ ) from human macrophages (Fig. 6b). To further probe the significance of ELR motif in binding of PfTyrRS to macrophages, we tested inhibition of PfTyrRS-macrophage interaction using peptides in which the wild-type PfTyrRS ELR peptide sequence (DELRV) was mutated residue-wise to DELAV, DAAAV and DELKL. Fluorescence-activated cell sorting (FACS)-based binding studies of PfTyrRS in presence of $100 \mathrm{nM}$ of these peptides showed a significant reduction in binding with only the DELRV peptide sequence (Fig. 6c). The DELEL peptide was less competent in competing whereas the other two peptides did not compete at all (Fig. 6c). Additionally, the PfTyrRS-triggered release of TNF- $\alpha$ and IL-6 from human macrophages was significantly reduced in presence of

Figure 5 | Effect of PfTyrRS on mouse macrophages. (a) Cytokines levels were measured using BioPlex kit (Bio-Rad) and the secretion profiles of TNF- $\alpha$ and IL- 6 using mouse macrophages were measured at different time points. Media alone, PfDTD/PfSerRS enzymes and ELR-AAA mutant of PfTyrRS were used as negative controls. LPS was used as positive control in all experiments. (b) Identical experiment as in (a) except that the human macrophage cell line ThP1 was used. This experiment has the extra negative control of PfSerRS. (c) Secretion profiles of extra cytokines (IL-1, IL-10 and IL-12) that are secreted from human macrophages on exposure to PfTyrRS. (d) Dose-dependent increase in secretion of TNF- $\alpha$ and IL- 6 when increasing concentrations of PfTyrRS were used with human macrophages. (e) Cytokine secretion profiles using P. falciparum culture supernatants alone (Sup), supernatants incubated with anti-TyrRS antibody (Sup $+\alpha$ TyrRS), parasite culture supernantant-immunoprecipitated PfTyrRS (PD-TyrRS) and finally parasite culture supernantant -immunoprecipitated PfTyrRS with its antibody (PD-TyrRS $+\alpha$ TyrRS). TNF- $\alpha$ and IL- 6 secretion is clearly enhanced significantly when immunoprecipitated PfTyrRS (PDTyrRS) is used. Triggering activity of PfTyrRS (PD-TyrRS) is blocked when pre-incubated with anti-PfTyrRS antibodies, indicating specific activation of macrophages with native PfTyrRS (PD-TyrRS). Experiments were repeated three times and error bars indicate standard deviation. Tukey's test was performed and $P$-values are shown such that the end points of the lines below $P$-value represent the datasets that were compared. 
a

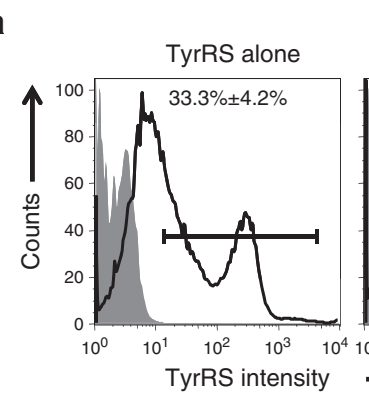

TyrRS tanti-CXCR1

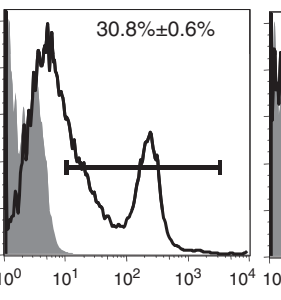

TyrRS +anti-CXCR2

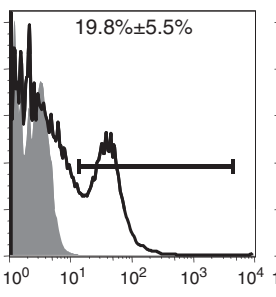

TyrRS +anti-CXCR1+2
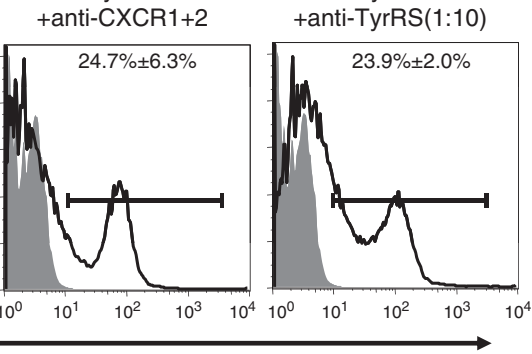

b
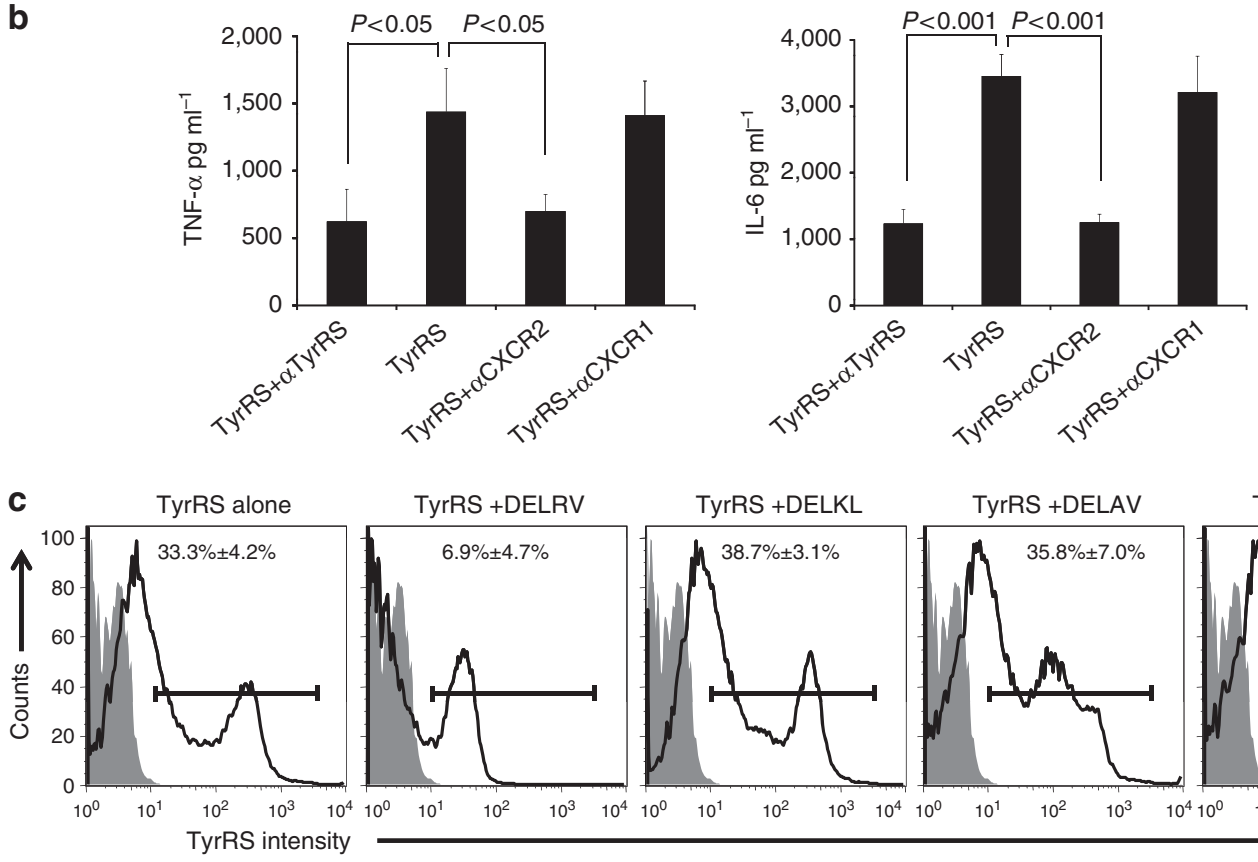

TyrRS +DAAAV

d
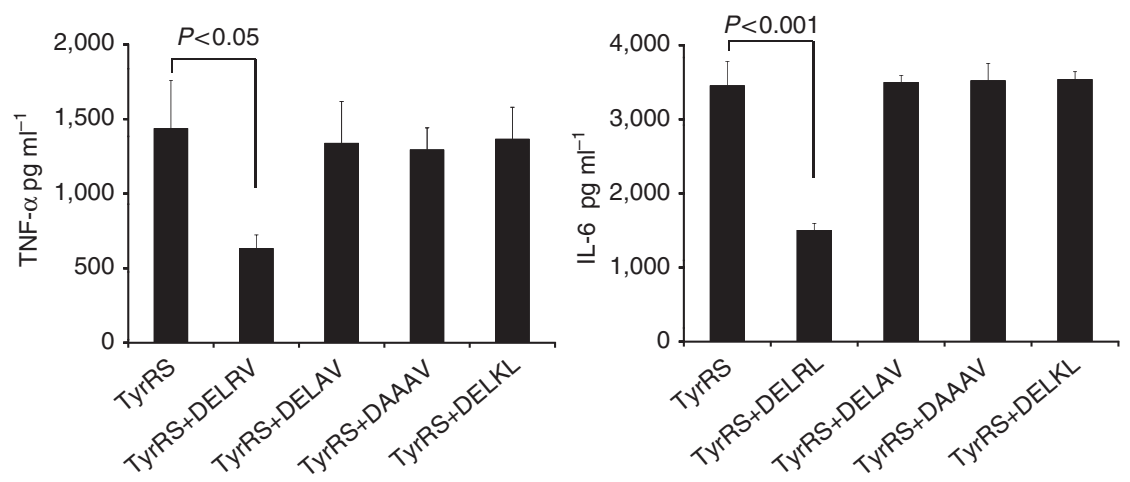

Figure 6 | Potential PfTyrRS receptor on human macrophages. (a) FACS analysis of PfTyrRS binding to human macrophages. PfTyrRS binding to macrophages is reduced when macrophages had been pre-incubated anti-CXCR2 or with anti-PfTyrRS antibodies. Binding was not reduced significantly with anti-CXCR1 antibodies. Overlay histograms show binding of PfTyrRS to the surface of human macrophages (black line) with unstained controls (grey solid) as background. (b) Cytokine secretion assays for TNF- $\alpha$ and IL- 6 using human macrophages and anti-CXCR1, anti-CXCR2 and anti-PfTyrRS antibodies. Secretion of these pro-inflammatory cytokines is reduced when anti-CXCR2 and anti-PfTyrRS antibodies were pre-incubated with human macrophages. (c) FACS analysis of PfTyrRS binding to human macrophages using four different ELR-mutant peptides in competition assays. Overlay histograms show PfTyrRS surface staining (black line) with unstained control (grey solid) as background. (d) Cytokine secretion assays for TNF- $\alpha$ and IL- 6 using human macrophages and various ELR-mutant peptides. No significant difference was observed for mutant peptides in these assays. Experiments were repeated three times and the error bars indicate the standard deviation. Tukey's test was performed and $P$-values are shown such that the end points of the lines below $P$-value represent the datasets that were compared.

DELRV peptide (Tukey's test $-P<0.05)$ (Fig. 6d). These data, along with our observation that the 'ELR/AAA' PfTyrRS mutant does not bind to human macrophage cells (Fig. 4c,d), strongly highlight a role for ELR motif in PfTyrRS interactions with human macrophages. We therefore conclude that ELR-motif-based PfTyrRS binding likely occurs using specific receptors on human macrophages, and that this binding leads to very specific host immune system activation.

PfTyrRS-stimulated macrophage upregulate ICAM-1 and VCAM-1. $P$. falciparum cytoadherence enables parasite seques- 

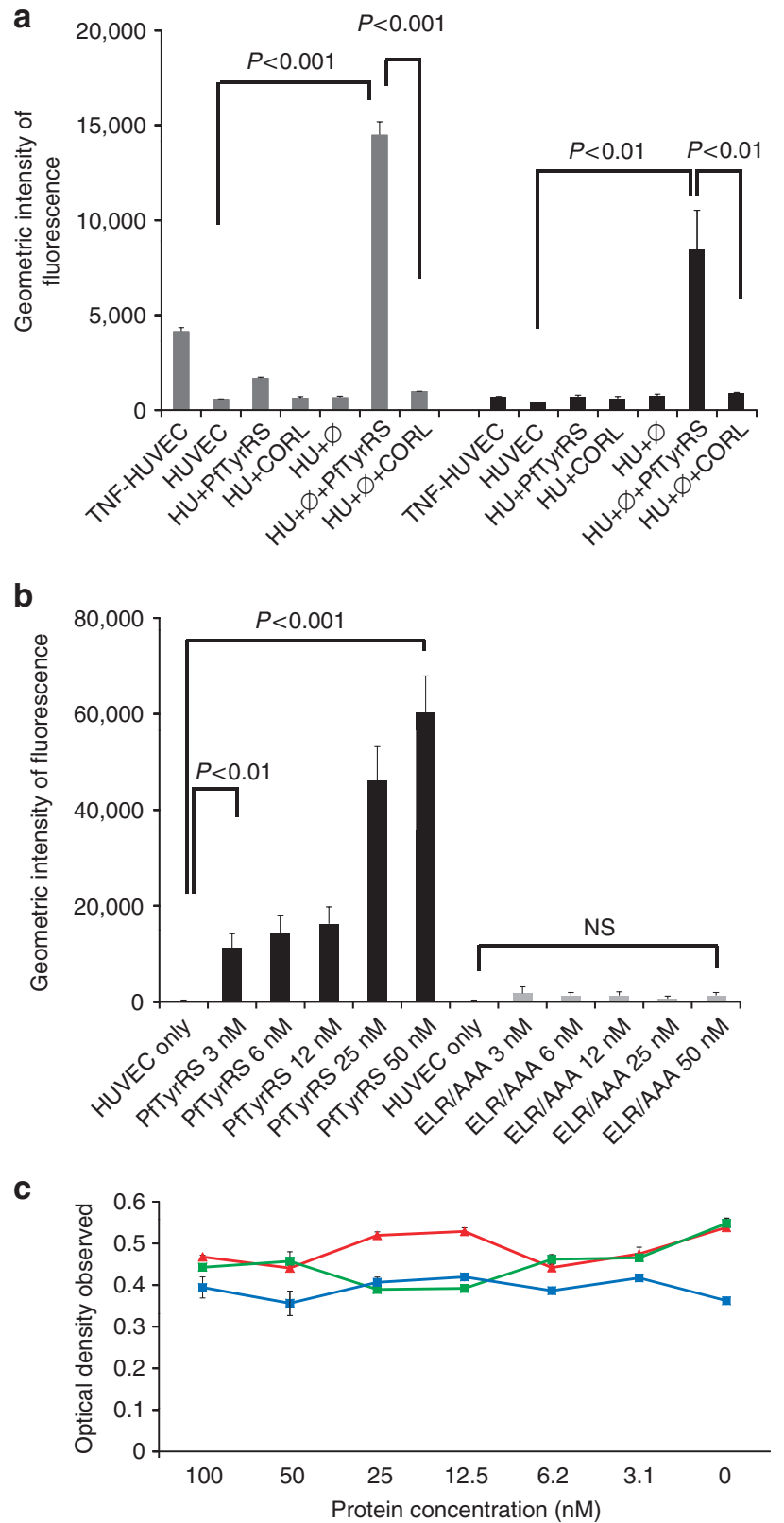

Figure 7 | PfTyrRS stimulation of macrophages and subsequent upregulation of endothelial receptors ICAM-1 and VCAM-1. (a) HUVEC cells $(\mathrm{Hu})$ were incubated with PfTyrRS alone, PFDTD (negative control protein (CORL), macrophage cells $(\phi)$ and TNF- $\alpha$. Culture supernatants of PfTyrRS-induced macrophages and PfDTD-induced macrophages were added to HUVEC cell lines. Expression of ICAM-1 and VCAM-1 was measured by FACS using specific conjugated antibodies. Grey bars (left) represent VCAM-1 expression and black bars (right) represent ICAM-1 expression. (b) PfTyrRS-induced dose-dependent increase in the expression of ICAM-1. No upregulation of ICAM-1 was observed on addition of ELR/AAA PfTyrRS mutant to endothelial cell lines. Bars in black represent PfTyrRS treated samples, whereas bars in grey were the samples treated with ELR/AAA mutant protein (c) MTT cytotoxicity assay with dose-dependent response using PfTyrRS (red line), ELR/AAA mutant (green line), and control (only cells in blue line) on HUVEC cells. No decrease in cell viability was observed (Tukey's test $-P>0.05$ ). The optical density on $y$ axis in (c) represents cell survival in an MTT assay. Experiments were repeated three times and the error bars represent standard deviation. Tukey's test was performed and $P$-values are shown such that the end points of the lines below $P$-value represent the datasets that were compared. NS, not significant. tration in host tissues, allowing these parasites to avoid splenic clearance which involves degradation and clearing of infected RBCs by the spleen ${ }^{34-40}$. In severe malaria episodes, an increase in the levels of TNF- $\alpha$ is coupled with enhanced expression of endothelial cell receptors such as ICAM-1 (intercellular adhesion molecule-1), VCAM-1 (vascular cell adhesion molecule-1) and E-selectin ${ }^{34-40}$. We therefore tested whether recombinant PfTyrRS could modulate expression of endothelial receptors in a static cytoadhesion model assay system. For this 'triggering' to work, PfTyrRS had to first initiate enhanced secretion of cytokines like TNF- $\alpha$ from macrophages, and then the increased levels of secreted cytokines would have to induce overexpression of endothelial receptors like ICAM-1 and VCAM-1. As our experimental data show, this is indeed what PfTyrRS was able to achieve (Fig. 7). When HUVECs (primary human umbilical vein endothelial cells) were co-cultured with culture supernatants of PfTyrRS-activated macrophages, we found strong upregulation of both ICAM-1 and VCAM-1 receptors (Tukey's test $-P<0.05$ ) (Fig. 7a). A PfTyrRS-induced dose-dependent increase in expression of ICAM-1 was also evident (Fig. 7b). These effects were not due to cell toxicity of PfTyrRS on HUVECs (Fig. 7c). These experiments therefore provide fundamental molecular insights and also a tool to dissect the molecular basis of cytoadherence using PfTyrRS as a 'trigger'. Increased levels of circulating cytokines in malaria infected individuals may activate the host immune system to check or control very high parasitemia levels, which may prove lethal both to pathogen and host.

\section{Discussion}

One of the key pathogenic mechanisms in the virulence of P. falciparum is the phenomenon of cytoadherence, attributed to increased adhesion of infected erythrocytes to blood capillaries ${ }^{34-42}$. In particular, cerebral and placental malaria seem to be dependent on crucial protein-protein interactions that occur between a parasite virulence factors and the endothelial/placental receptors ${ }^{34-42}$. The host immune system is hijacked by parasite-derived factors to assist in cytoadherence, for example, by cytokine-induced increased expression of a range of adhesion molecules ${ }^{16,18-25}$. Numerous field reports on human malaria and mouse model studies have shown that malaria infection alters host cytokine profiles by modulating dendritic cells and macrophages ${ }^{16,18-25}$. This is presumably done to aid in parasite survival and transmission. Elevated cytokine levels have a variety of physiological effects, generally to the detriment of the hosts ${ }^{16,34-42}$. In fact, malaria as a disease has been looked up on as a manifestation of enhanced pro-inflammatory cytokine production in host $\mathrm{t}^{16,34-42}$. For example, the clinical manifestations of severe malaria can be correlated with induction of strong pro-inflammatory responses in which plasma levels of circulating TNF- $\alpha$, IFN- $\gamma$ and IL-6 increase significantly ${ }^{19-21}$. Pathways that propel host immune cell activation in malaria remain mysterious in context of parasitederived protein antigens. The interaction of latter with macrophages and dendritic cells must occur before cytokine induction. So far, studies have identified several such molecular agents ${ }^{2-22}$, but, to our knowledge, no parasite-secreted housekeeping enzyme has been attributed with the ability to trigger enhanced secretion of cytokines like TNF- $\alpha$ and IL- 6 . In this context, we find it very interesting that the levels of TNF- $\alpha$ secreted from immune cells reported in previous studies $^{22-25}$ are similar to or lower than those we report with PfTyrRS. A comparison of secreted TNF- $\alpha$ levels $\left(\mathrm{pg} \mathrm{ml}^{-1}\right)$ is illuminatingusing $100 \mathrm{nM}$ PfTyrRS: 1,000-1,300 $\mathrm{pg} \mathrm{ml}^{-1}, 50-400 \mathrm{nM}$ GPIs; $800-$ $1,300 \mathrm{pg} \mathrm{ml}^{-1}, \sim \mu \mathrm{g} \mathrm{ml}^{-1}$ DNA-protein complexes; $500-600 \mathrm{pg} \mathrm{ml}^{-1}$ and $30-100 \mu \mathrm{M}$ hemozoin; $400-600 \mathrm{pg} \mathrm{ml}^{-1}$, $\sim 500,000$ microparticles; $150-200 \mathrm{pg} \mathrm{ml}^{-1}$. This comparison indicates importance of PfTyrRS as a trigger for excitation of pro-inflammatory cytokine release from host immune cells.

Our corroborative confocal microscopy data not only reveals decoration of host macrophage surfaces with PfTyrRS but also its 
internalization. We propose that, as a result of $P f$ TyrRS-macrophage interactions, significantly higher amounts of pro-inflammatory cytokines like TNF- $\alpha$, IL- 1 and IL- 6 are released. Elevated cytokine levels can have severe effects in hosts and severe malarial anemia (SMA) provides an excellent example of this ${ }^{45}$. Increased circulating TNF- $\alpha$ inhibits erythroblast differentiation and dampens erythropoiesis resulting in worsening SMA symptoms ${ }^{16-21,45}$. Another outcome of higher levels of circulating TNF- $\alpha$ in infected malaria patients is the increase in expression levels of certain endothelial receptors like ICAM-1, VCAM-1 and E-selectin that contribute to cytoadherence in severe malaria ${ }^{39-42}$. Our data based on co-incubation of three components-purified PfTyrRS macrophages and endothelial cells, now provides a system that can be further dissected to investigate molecular and cellular interactions that occur during SMA and cytoadherence. This work therefore provides clues to the possibility of $P f$ TyrRS acting as a malarial toxin that can induce cytokine-mediated disease.

Aminoacyl-tRNA synthetases have a vital role in translating the genetic code. Numerous studies have shown that members of this enzyme family are quite adept at 'moonlighting' in terms of possessing extra capabilities that widen their biological attributes ${ }^{5-8}$. Studies have indicated that human tyrosyl-, tryptophanyl- and lysyl-tRNA synthetases can be secreted extracellularly and can mimic cytokines ${ }^{5-8}$. For these extra roles, the human TyrRS and TrpRS require either proteolytic cleavage or alternate splicing to become active $e^{5-9}$, whereas LysRS can act like a cytokine without processing ${ }^{44}$. In this light, this is the first report of a human pathogen tRNA synthetase that can also perform moonlighting activity of inducing host immune system to release proinflammatory cytokines. In summary, this study suggests that $P f$ TyrRS is secreted from the parasite and deposited proximal to $\mathrm{iRBC}$ membrane (despite lack of a 'PEXEL' export element ${ }^{43}$ ). PfTyrRS egress occurs during trophozoite and schizont stages, before iRBC rupture. On iRBC lysis, PfTyrRS is released into blood plasma, where it is potentially free to interact with host cells. We show that $P f$ TyrRS interacts with specific receptor(s) on host macrophages, and that this event is largely mediated by the ELR motif present in $P f$ TyrRS. Finally, we have determined the three-dimensional structure of $P f$ TyrRS in complex with Tyr-AMP, and provide detailed structural comparisons with host counterpart of PfTyrRS. This work therefore not only provides a structural framework for using $P f$ TyrRS-YAP complex structure as a starting point for structure-based drug discovery, but also lays foundation for probing key immunological questions in malaria. The twin abilities of malaria parasite tyrosyl-tRNA synthetase-as a housekeeping protein translation enzyme and as a trigger for pro-inflammatory cytokine release in hosts-highlight this protein as a target for discovery of novel antiparasitic strategies.

\section{Methods}

Animal experiments. Animal experiments were performed in accordance with Institutional Animal Ethics Committee guidelines approved by Department of Biotechnology, Govt of India. This project was approved in a meeting held on 28 October 2009 at ICGEB (approval ID: ICGEB/IAEC/MAL-50). All mice used were killed ethically by asphyxiation in carbon dioxide according to regulatory body instructions.

Synthetic tRNA preparation and aminoacylation assay. Synthetic Plasmodium falciparum tRNA clones were obtained according to Sampson and Uhlenbeck ${ }^{46}$ by assembling up to 6 oligonucleotides and ligation between HindIII and BamHI sites into pUC19 vector. The tRNAs were obtained by in vitro transcription using T7 RNA polymerase according to standard protocols. In vitro tRNA aminoacylation assays were performed at $37^{\circ} \mathrm{C}$ in $50 \mathrm{mM}$ Tris- $\mathrm{HCl} \mathrm{pH} \mathrm{7.6,50} \mathrm{mM} \mathrm{KCl}$, $25 \mathrm{mM} \mathrm{MgCl}_{2}, 5 \mathrm{mM}$ ATP, $0.1 \mathrm{mg} \mathrm{ml}^{-1} \mathrm{BSA}, 1 \mathrm{mM}$ DTT, $20 \mu \mathrm{M}$ tyrosine, 0.5 $\mathrm{mCi} \mathrm{ml}^{-1} \mathrm{~L}-[3,5-3 \mathrm{H}]$ tyrosine and $0.5 \mu \mathrm{M}$ PfTyrRS as reported ${ }^{47}$. Reaction was initiated by addition of pure enzyme and samples of $20 \mu \mathrm{l}$ were spotted onto Whatman $3 \mathrm{mM}$ discs at varying time intervals (usually $2 \mathrm{~min}$ ). Radioactivity (corresponding to amino acid ligated to tRNA substrate) was measured by liquid scintillation.

Protein expression, purification and crystallization. TyrRS gene (MAL8P1.125). from P. falciparum was PCR amplified from complementary DNA and cloned into PQE30 vector (Qiagen). Four hours post -IPTG (isopropyl 1-thio-Dgalactopyranoside, $0.5 \mathrm{mM}$ ) induction, the bacterial cell pellet was suspended in buffer containing $50 \mathrm{mM}$ Tris- $\mathrm{HCl}, 500 \mathrm{mM} \mathrm{NaCl}, 50 \mathrm{mM}$ imidazole, $\mathrm{pH} 8.0$ and protease inhibitor cocktail. Protein was purified using Ni-NTA beads (Qiagen) and gel filtration chromatography. Pure, dimeric $P f$ TyrRS was concentrated and buffer exchanged into $25 \mathrm{mM}$ Tris- $\mathrm{HCl} \mathrm{pH} \mathrm{8.0,150} \mathrm{mM} \mathrm{NaCl}$ and $10 \mathrm{mM}$ BME. Diffraction quality crystals using His-tag-free $P f$ TyrRS were grown by hanging drop vapour diffusion method at $20^{\circ} \mathrm{C}$. The droplet size was $1 \mu \mathrm{l}$ of protein $\left(30 \mathrm{mg} \mathrm{ml}^{-}\right.$ in $25 \mathrm{mM}$ Tris $\mathrm{pH}$ 8.0, $100 \mathrm{mM} \mathrm{NaCl}, 10 \mathrm{mM}$ BME, $10 \mathrm{mM} \mathrm{MgCl}, 10 \mathrm{mM}$ ATP and $2 \mathrm{mM} \mathrm{L}$-tyrosine) with $1 \mu \mathrm{l}$ of reservoir solution equilibrated over $200 \mu \mathrm{l}$ of $2.4 \mathrm{M}$ sodium malonate at $\mathrm{pH} 6.0$.

Data collection and structure determination. A single crystal was transferred to cryoprotectant reagent (paratone oil) for a short period (5-10 s) before freezing in a stream of cooled nitrogen gas. Data were collected at $100 \mathrm{~K}$ using $\mathrm{Cu} \mathrm{K} \alpha$ radiation $(\lambda=1.54 \AA)$ on a MAR345 image-plate detector mounted on a Rigaku MicroMax007 rotating-anode $\mathrm{X}$-ray generator. A total of 700 frames were collected in $1^{\circ}$ oscillation steps with $5 \mathrm{~min}$ exposure per frame, and data were processed with HKL2000 (ref. 48). The structure was solved by molecular replacement in MOLREP $^{49}$ using cytoplasmic mini-HsTyrRS (PDB: 1N3L) as a starting model. Iterated manual model building and refinement was done using $\mathrm{COOT}^{50}$ and phenix. refine $^{51}$. The structural representations in this paper were prepared with Chimera ${ }^{52}$ and PyMOL (http://www.pymol.org).

Cell-binding assays. For in vitro localization experiments, synchronous parasite cultures of $P$. falciparum 3D7 were washed with PBS, fixed in solution using 4\% paraformaldehyde and $0.0075 \%$ glutraldehyde for $20 \mathrm{~min}$, permeablized with $0.1 \%$ Triton X-100 for $10 \mathrm{~min}$ and then treated for $10 \mathrm{~min}$ with $0.1 \mathrm{mg} \mathrm{ml}^{-1}$ sodium borohydride in PBS. Each step was followed by a brief PBS wash ${ }^{53}$. Cells were blocked in 3\% BSA, PBS for $1 \mathrm{~h}$, washed in PBS and incubated overnight with protein-A column purified rabbit anti-PfTyrRS antibodies (1:150 dilution in PBS containing $3 \% \mathrm{BSA}$ ) at $4^{\circ} \mathrm{C}$. After three washes ( $10 \mathrm{~min}$ each) with PBS, the cells were incubated with Alexa Fluor ${ }^{488}$-conjugated anti-rabbit secondary antibody ( $1: 250$ dilution in PBS containing 3\% BSA) for $2 \mathrm{~h}$ at room temperature and allowed to settle onto poly-D-lysine $\left(100 \mathrm{mg} \mathrm{m}^{-1}\right)$-coated coverslips. These were then washed three times in PBS and mounted in antifade with DAPI (Molecular Probes). Spleen cells were purified from P. yoelii-infected mice and PBMCs were isolated from both mouse and human blood as described earlier ${ }^{54}$. For the in vivo experiment, C57BL/6 mice were infected with $\sim 1$ million P. yoelii (17XNL strain) in the form of parasitized RBC's intraperitoneally. Fifteen days after infection, the spleens were collected from infected and age-and-sex matched uninfected control mice. Spleens were crushed and lymphocytes were isolated and stained for flow cytometry by standard procedures. The cells were fixed with Cytofix (BD Biosciences) and permeabilized with 1X Cytoperm (BD Biosciences) for intracellular TyrRS staining. The data was acquired on BD FACS Canto II (BD Biosciences) and analysed on by FlowJo software (Treestar). Human PBMCs were isolated using $\mathrm{BD}$ Vacutainer $\mathrm{CPT}$ tubes (BD Biosciences) by centrifugation at $1,600 \mathrm{~g}$ per $25 \mathrm{~min} / \mathrm{RT} / \mathrm{no}$ brake. The cells were thoroughly washed and resuspended in complete RPMI containing $10 \%$ FCS in a 24-well plate, and $100 \mathrm{nM}$ purified $P f$ TyrRS protein was added for $3 \mathrm{~h}$ at $37^{\circ} \mathrm{C}\left(95 \%\right.$ humidity, $\left.5 \% \mathrm{CO}_{2}\right)$. Analytical flow cytometry was performed by direct staining for specific populations of immune cells with fluorescently conjugated antibodies and biotinconjugated anti-PfTyrRS antibody (1:150 dilution in PBS). The data obtained on BD FACS Canto II (BD Biosciences) was analysed using FlowJo software (Treestar) In vitro binding of $P f$ TyrRS to various immune cells was performed by FACS using standard protocols. Dendritic cells and macrophage ThP1 cells were cultured on coverslips in 24-well plates (Gibson) and maintained in DMEM medium containing 20\% (v/v) FBS and 1\% antibiotics (Gibco). PfTyrRS and other control proteins were incubated with cells at several concentrations for $5 \mathrm{~min}$ to $2 \mathrm{~h}$ at $4{ }^{\circ} \mathrm{C}$. Cells were washed twice in PBS and incubated with 2\% formaldehyde (Sigma) for $15 \mathrm{~min}$ and followed by incubation for $10 \mathrm{~min}$ with $0.1 \%$ saponin (Sigma). Primary and secondary antibodies were added as per manufacturer's instructions (Molecular Probes). Fluorescence microscopy was performed on Nikon A1R confocal laser scanning microscope.

Western blots. P. falciparum (schizont stage) -infected human erythrocytes $(1.5 \times 107$ parasites per $\mathrm{ml})$ were enriched and parasites were cultured for $6-10 \mathrm{~h}$ to allow the maturation of schizonts, release of merozoites and their material ${ }^{54}$. Culture supernatant was collected in the presence of protease inhibitor cocktail after two rounds of centrifugation of $1,000 \mathrm{~g}$ for $15 \mathrm{~min}$ followed by $10,000 \mathrm{~g}$ for $20 \mathrm{~min}$ before freezing at $-70^{\circ} \mathrm{C}$. Culture supernatant was subsequently used for western blotting using different antibodies against PfDTD (dilution, 1:500), $P f$ falcipain 2 (dilution, (1:1,000), PfMSP-1 (dilution, 1:1,000) and PfTyrRS (dilution, 1:500).

Cytokine secretion assays. Raw-264.7. cells, J774 murine cells, Dendritic cells (isolated from femur bone marrow of the mice C57BL/6) and human THP1 macrophage cells $\left(3 \times 10^{4}\right)$ were cultured in RPMI medium with $10 \%$ FBS and $1 \%$ antibiotics in 24 -well format plates. Proteins were passed through polymyxin 
beads (Sigma) before assays. LPS, PfTyrRS and control proteins were added to the medium at different concentrations. $100 \mu \mathrm{l}$ of culture medium was collected and centrifuged after every 4, 24 and $48 \mathrm{~h}$. Cytokines in the culture supernatant were detected by a Luminex microbead-based multiplexed assay (Bio-Plex manager 5.0) using commercially available kits according to the manufacturer's protocol (Bio-Plex, Bio-Rad). All proteins were tested for endotoxin contaminations and passed through polymyxin beads (Sigma). LAL (Limulus Amebocyte Lysate) assay was performed to confirm that the proteins were endotoxin-free before each assay.

PfTyrRS interaction with human macrophages. Cultured human macrophages cells were mixed with PfTyrRS in absence or presence of different antibodies, namely anti-PfTyrRS, anti-CXCR1 and anti-CXCR2. Binding of PfTyrRS was tested using standard FACS protocols. In related experiments, cytokine profiling of these human macrophages was also done, either in absence or presence of antibodies used above. Cytokines released in culture supernatants were detected by a Luminex microbead-based multiplexed assay (Bio-Plex manager 5.0) using commercially available kits and according to the manufacturer's protocol (Bio-Plex, Bio-Rad). Similar experiments were performed using various wild-type and mutant ELRbased peptides. Human macrophages (ThP1 cells) were incubated with $100 \mathrm{nM}$ of these peptides before addition of PfTyrRS. FACS and cytokine profiling were again done on all these samples as for the antibody treatment experiments.

Upregulation of endothelial cell receptors. Monocytes were obtained from buffy coat by Lymphoprep (Axis-Shield) gradient centrifugation, followed by adhesionmediated purification on tissue culture plastic. The cells were cultured for 3 days in RPMI-164 medium (Sigma) supplemented with $2 \mathrm{mM}$ L-glutamine and $10 \%$ FBS to differentiate into macrophage. The recombinant $P f$ TyrRS or control proteins were added at a concentration of $1 \mu \mathrm{ml}^{-1}$ on day 3 , and cells were cultured continuously for 3 days. The culture supernatant was collected and centrifuged at $13,000 \mathrm{~g}$ for $10 \mathrm{~min}$ to remove any debris; the supernatant was used for co-culture with endothelium. Primary pooled human umbilical vein endothelial cells (HUVEC) were obtained from Promocell). Cells were maintained in complete growth medium supplied by Promocell, according to the company's instructions, To reduce variation, only cells at passage five to six were used. HUVEC were grown on $1 \%$ gelatin (Sigma) coated on 24 -well plates with appropriate growth medium, until confluent. Then, the culture medium was replaced with either fresh medium alone, medium containing recombinant $P f$ TyrRS or control protein (added at concentration $100 \mathrm{nM}$ ) or macrophage culture supernatants. These were incubated at $37^{\circ} \mathrm{C}$ in $5 \% \mathrm{CO}_{2}$ for $16 \mathrm{~h}$. Endothelial cell markers were measured by fluorescence-activated cell sorting (FACScan; Becton Dickinson). Specific fluorescenceconjugated antibodies, including APC Mouse anti-human CD54 (ICAM-1) and PE Mouse anti-human CD106 (VCAM-1) (Becton Dickinson), were used in this study. Nonspecific fluorescence was assessed using corresponding isotype control antibodies. The expression of endothelial surface proteins ICAM-1 and VCAM-1 was indicated by geometric mean of the fluorescence intensity. PfTyrRS was subsequently used in a dose-dependent experiment for testing increased expression of ICAM-1, using the protocols described above.

PfTyrRS-GFP microscopy. The gene was amplified with genomic DNA of P. falciparum using following primers: forward-5'-CTCGAGATGATAATAT TAAAGGTTTTATTTA- ${ }^{\prime}$ ' reverse-5'-GGTACCTTTTATTATTATAGAATAAC TTGTTC- $3^{\prime}$ and cloned into pGEM-T vector (Promega). The insert was released from pGEMT and cloned between XhoI and KpnI in pGlux vector (Amersham). Transformations and clone propagation was done in $\mathrm{Dh} 5 \alpha$ cells. Transfection of vectors into the parasite was done, as described earlier ${ }^{55}$. GFP-expressing infected erythrocytes were studied live or fixed at ambient temperature. Cells were viewed with a UPlanSApo $\times 1001.4$ oil objective on a Olympus IX81 Live Cell Imaging Inverted Microscope equipped with an Olympus F-View camera and primarily processed with analySIS LS Research software package. Captured images were then further processed using Photoshop and ImageJ. Pictures were adjusted to gain optimal contrast to visualize features of interest.

MTT assays. HUVEC cells at passage 5 were seeded at a concentration of $2 \times 10^{4}$ cells per well (in $100 \mu \mathrm{l}$ ) and allowed to attach for $24 \mathrm{~h}$ on $1 \%$ gelatine (Sigma) coated on 96-well plates in complete growth medium under standard conditions ( $5 \% \mathrm{CO}_{2}, 95 \%$ humidity, $37^{\circ} \mathrm{C}$ ). Then, the cells were exposed to $P f$ TyrRS at the concentrations indicated along with mutant $P f$ TyrRS or HUVEC alone. The exposure time was 24 hours. Afterwards, the cell culture medium was withdrawn and in each well $10 \mu$ MTT [3-(4,5-dimethylthiazol-2-yl)-2,5-diphenyl tetrazolium bromide, Sigma] stock solution $\left(5 \mathrm{mg} \mathrm{ml}^{-1}\right)$ was added, followed by an incubation period of $4 \mathrm{~h}$. Afterwards, $100 \mu \mathrm{l}$ per well stop solution (40 g SDS per $200 \mathrm{ml} 50 \%$ DMF (NN-Dimethyformide, Sigma)) was added, followed by an incubation step overnight $\left(37^{\circ} \mathrm{C}\right)$. MTT metabolization as parameter for metabolic activity was recorded spectrophotometrically by Varioskan at $570 \mathrm{~nm}$

Statistical analyses. Data are derived from multiple independent experiments done in triplicates. Statistical analyses were conducted using SPSS software and values are presented as mean \pm s.d. Significant differences between groups were determined by ANOVA followed by Tukey's multiple comparison test (SPSS software). A value of $P<0.05$ was accepted as an indication of statistical significance.

\section{References}

1. Ibba, M. \& Söll, D. Aminoacyl-tRNA synthesis. Annu. Rev. Biochem. 69, 617-650 (2000)

2. Ibba, M. \& Söll, D. The renaissance of aminoacyl-tRNA synthesis. EMBO Rep. 2, 382-387 (2001).

3. Eriani, G., Delarue, M., Poch, O., Gangloff, J. \& Moras, D. Partition of tRNA synthetases into two classes based on mutually exclusive sets of sequence motifs. Nature 347, 203-206 (1990).

4. Burbaum, J. J. \& Schimmel, P. Structural relationships and the classification of aminoacyl-tRNA synthetases. J. Biol. Chem. 266, 16965-16968 (1991).

5. Brown, M. V., Reader, J. S. \& Tzima, E. Mammalian aminoacyl-tRNA synthetases: cell signaling functions of the protein translation machinery. Vascul. Pharmacol. 52, 21-26 (2010).

6. Bhatt, T. K. et al. A genomic glimpse of aminoacyl-tRNA synthetases in malaria parasite Plasmodium falciparum. BMC Genomics 10, 644 (2009).

7. Guo, M., Yang, X. L. \& Schimmel, P. New functions of aminoacyl-tRNA synthetases beyond translation. Nat. Rev. Mol. Cell Biol. 11, 668-674 (2010).

8. Guo, M., Schimmel, P. \& Yang, X. L. Functional expansion of human tRNA synthetases achieved by structural inventions. FEBS Lett. 584, 434-442 (2010).

9. Wakasugi, K. \& Schimmel, P. Two distinct cytokines released from a human aminoacyl-tRNA synthetase. Science 284, 147-151 (1999).

10. Wakasugi, K., Slike, B. M., Hood, J., Ewalt, K. L., Cheresh, D. A. \& Schimmel, P. Induction of angiogenesis by a fragment of human tyrosyl-tRNA synthetase. J. Biol. Chem. 277, 20124-20126 (2002).

11. Yang, X. L. et al. Gain-of-function mutational activation of human tRNA synthetase procytokine. Chem. Biol. 14, 1323-1333 (2007).

12. Kapoor, M., Otero, F. J., Slike, B. M., Ewalt, K. L. \& Yang, X. L. Mutational separation of aminoacylation and cytokine activities of human tyrosyl-tRNA synthetase. Chem. Biol. 16, 531-539 (2009).

13. Yang, X. L., Skene, R. J., McRee, D. E. \& Schimmel, P. Crystal structure of a human aminoacyl-tRNA synthetase cytokine. Proc. Natl Acad. Sci. USA 99, 15369-15374 (2002).

14. World Health Organization (2009) WHO World malaria report, http://www. who.int/malaria/world_malaria_report_2009/en/index.html.

15. Milner, D. A. Jr. Rethinking cerebral malaria pathology. Curr. Opin. Infect. Dis. 23, 456-463 (2010).

16. Clark, I. A., Budd, A. C., Alleva, L. M. \& Cowden, W. B. Human malaria disease: a consequence of inflammatory cytokine release. Malaria J. 5, 85 (2006).

17. Riley, E. M., Wahl, S., Perkins, D. J. \& Schofield, L. Regulating immunity to malaria. Parasite Immunol. 28, 35-49 (2006).

18. Corrigan, R. A. \& Rowe, J. A. Strain variation in early innate cytokine induction by Plasmodium falciparum. Parasite Immunol. 32, 512-527 (2010).

19. Robinson, L. J. et al. Cellular tumor necrosis factor, gamma interferon, and interleukin- 6 responses as correlates of immunity and risk of clinical Plasmodium falciparum malaria in children from Papua New Guinea. Infect. Immun. 77, 3033-3043 (2009).

20. Dodoo, D., Omer, F. M., Todd, J., Akanmori, B. D., Koram, K. A. \& Riley, E. M. Absolute levels and ratios of proinflammatory and anti-inflammatory cytokine production in vitro predict clinical immunity to Plasmodium falciparum malaria. J. Infect. Dis. 185, 971-979 (2002).

21. Brustoski, K. et al. IFN- $\gamma$ and IL-10 mediate parasite-specific immune responses of cord blood cells induced by pregnancy-associated Plasmodium falciparum malaria. J. Immunol. 174, 1738-1745 (2005).

22. Coban, C. et al. Toll-like receptor 9 mediates innate immune activation by the malaria pigment hemozoin. J. Exp. Med. 201, 19-25 (2005).

23. Krishnegowda, G. et al. Induction of proinflammatory responses in macrophages by the glycosylphosphatidylinositols of Plasmodium falciparum: cell signaling receptors, glycosylphosphatidylinositol (GPI) structural requirement, and regulation of GPI activity. J. Biol. Chem. 280, 8606-8616 (2005).

24. Couper, K. N. et al. Parasite-derived plasma microparticles contribute significantly to malaria infection-induced inflammation through potent macrophage stimulation. PLoS Pathog. 6, e1000744 (2010).

25. Wu, X., Gowda, N. M., Kumar, S. \& Gowda, D. C. Protein-DNA complex is the exclusive malaria parasite component that activates dendritic cells and triggers innate immune responses. J. Immunol. 184, 4338-4348 (2010).

26. Fechter, P., Rudinger-Thirion, J., Théobald-Dietrich, A. \& Giegé, R. Identity of tRNA for yeast tyrosyl-tRNA synthetase: tyrosylation is more sensitive to identity nucleotides than to structural features. Biochemistry 39, 1725-1733 (2000)

27. Quinn, C. L., Tao, N. \& Schimmel, P. Species-specific microhelix aminoacylation by a eukaryotic pathogen tRNA synthetase dependent on a single base pair. Biochemistry 34, 12489-12495 (1995).

28. Tsunoda, M. et al. Structural basis for recognition of cognate tRNA by tyrosyl-tRNA synthetase from three kingdoms. Nucleic Acids Res. 35, 289-4300 (2007). 
29. Bonnefond, L. et al. Crystal structure of human mitochondrial tyrosyl-tRNA synthetase reveals common and idiosyncratic features. Structure 15, 1505-1516 (2007).

30. Zhao, X., Town, J. R., Li, F., Zhang, X., Cockcroft, D. W. \& Gordon, J. R. ELR-CXC chemokine receptor antagonism targets inflammatory responses at multiple levels. J. Immunol. 182, 3213-3222 (2009).

31. Gamo, F. J. et al. Thousands of chemical starting points for antimalarial lead identification. Nature 465, 305-310 (2010).

32. Bhatt, T. K., Yogavel, M., Wydau, S., Berwal, R. \& Sharma, A. Ligand-bound structures provide atomic snapshots for the catalytic mechanism of D-amino acid deacylase. J. Biol. Chem. 285, 5917-5930 (2010).

33. Yogavel, M., Khan, S., Bhatt, T. K. \& Sharma, A. Structure of D-tyrosyl-tRNA ${ }^{\text {Tyr }}$ deacylase using home-source $\mathrm{Cu} \mathrm{K} \alpha$ and moderate-quality iodide-SAD data: structural polymorphism and HEPES-bound enzyme states. Acta Crystallogr. D Biol. Crystallogr. 66, 584-592 (2010).

34. López, J. A. Malignant malaria and microangiopathies: merging mechanisms. Blood 115, 1317-1318 (2010).

35. Medana, I. M. \& Turner, G. D. Human cerebral malaria and the blood-brain barrier. Int. J. Parasitol. 36, 555-568 (2006)

36. Wilson, N. O. et al. Soluble factors from Plasmodium falciparum-infected erythrocytes induce apoptosis in human brain vascular endothelial and neuroglia cells. Mol. Biochem. Parasitol. 162, 172-176 (2008).

37. Combes, V., Coltel, N., Faille, D., Wassmer, S. C. \& Grau, G. E. Cerebral malaria: role of microparticles and platelets in alterations of the blood-brain barrier. Int. J. Parasitol. 36, 541-546 (2006).

38. Pasternak, N. D. \& Dzikowski, R. PfEMP1: an antigen that plays a key role in the pathogenicity and immune evasion of the malaria parasite Plasmodium falciparum. Int. J. Biochem. Cell Biol. 41, 14631466 (2009).

39. Tripathi, A. K., Sha, W., Shulaev, V., Stins, M. F. \& Sullivan, D. J. Jr. Plasmodium falciparum-infected erythrocytes induce NF-kappaB regulated inflammatory pathways in human cerebral endothelium. Blood 114, 4243-4252 (2009).

40. Stevenson, M. M. \& Urban, B. C. Antigen presentation and dendritic cell biology in malaria. Parasite Immunol. 28, 5-14 (2006).

41. Diouf, I. et al. IL-12 producing monocytes and IFN-gamma and TNF-alpha producing T-lymphocytes are increased in placentas infected by Plasmodium falciparum. J. Reprod. Immunol. 74, 152-162 (2007).

42. Francischetti, I. M., Seydel, K. B. \& Monteiro, R. Q. Blood coagulation, inflammation, and malaria. Microcirculation 15, 81-107 (2008).

43. Goldberg, D. E. \& Cowman, A. F. Moving in and renovating: exporting proteins from Plasmodium into host erythrocytes. Nat. Rev. Microbiol. 8, 617-621 (2010).

44. Park, S. G. et al. Human lysyl-tRNA synthetase is secreted to trigger proinflammatory response. Proc. Natl Acad. Sci. USA 102, 6356-6361 (2005).

45. Haldar, K. \& Mohandas, N. Malaria, erythrocytic infection, and anemia. Hematology Am. Soc. Hematol. Educ. Program 87-93 (2009)

46. Sampson, J. R. \& Uhlenbeck, O. C. Biochemical and physical characterization of an unmodified yeast phenylalanine transfer RNA transcribed in vitro. Proc. Natl Acad. Sci. USA 85, 1033-1037 (1988).

47. Schulman, L. H. \& Pelka, H. Anticodon loop size and sequence requirements for recognition of formylmethionine tRNA by methionyl-tRNA synthetase. Proc. Natl Acad. Sci. USA 80, 6755-6759 (1983).
48. Otwinowski, Z. \& Minor, W. Processing of X-ray diffraction data collected in oscillation mode. Methods Enzymol. 276, 307-326 (1997).

49. Vagin, A. \& Teplyakov, A. Molecular replacement with MOLREP. Acta Crystallogr. D Biol. Crystallogr. 66, 22-25 (2010)

50. Emsley, P. \& Cowtan, K. Coot: model-building tools for molecular graphics. Acta Crystallogr. D Biol. Crystallogr. 60, 2126-2132 (2004).

51. Adams, P. D. et al. PHENIX: building new software for automated crystallographic structure determination. Acta Crystallogr. D Biol. Crystallogr. 58, 1948-1954 (2002).

52. Pettersen, E. F. et al. UCSF Chimera--a visualization system for exploratory research and analysis. J. Comput. Chem. 25, 1605-1612 (2004).

53. Tonkin, C. J. et al. Localization of organellar proteins in Plasmodium falciparum using a novel set of transfection vectors and a new immunofluorescence fixation method. Mol. Biochem. Parasitol. 137, 13-21 (2004)

54. Haynes, J. D. et al. Receptor-like specificity of a Plasmodium knowlesi malaria protein that binds to Duffy antigen ligands on erythrocytes. J. Exp. Med. 167, 1873-1881 (1988).

55. Crabb, B. S. et al. Transfection of the human malaria parasite Plasmodium falciparum. Methods Mol. Biol. 270, 263-276 (2004).

\section{Acknowledgements}

We thank Monika Chugh for help with western blotting of culture supernatants. T.K.B. S.K., M.M.B. and A.S. are supported by Department of Biotechnology and CSIR fellowships. A.G.M. is an ARC Australian Research Fellow. This work in part was conducted under EU MEPHITIS project (Grant Agreement no: HEALTH-F3-2009-223024). Funding for research on malaria tRNA synthetases is provided by a DBT grant to the laboratory.

\section{Author contributions}

T.K.B., S.K., V.D. and M.Y. carried out protein studies, collected localization data, and performed structural analyses along with immunological experiments. T.K.B. S.K., M.Y., A.C. and A.S. analysed all data and wrote the manuscript. M.M.B. performed cloning, purified PfTyrRS and crystallized it. A.S. and A.C. helped with cell biology experiments. N.C. and R.P.L. performed aminoacylation assays. Y.W. and A.G.C. performed endothelial activation assays. A.G.M. and A.T.M. performed PfTyrRS-GFP microscopy.

\section{Additional information}

Acccession codes: Coordinates and structure factors for PfTyrRS have been deposited in the RCSB Protein Data Bank under accession code 3vgj.

Supplementary Information accompanies this paper at http://www.nature.com/ naturecommunications

Competing financial interests: The authors declare no competing financial interests.

Reprints and permission information is available online at http://npg.nature.com/ reprintsandpermissions/

How to cite this article: Bhatt, T. K. et al. Malaria parasite tyrosyl-tRNA synthetase secretion triggers pro-inflammatory responses. Nat. Commun. 2:530 doi: 10.1038/1522 (2011). 